\title{
Detached eddy simulation of compressible flow with rapid expanded divergent contour
}

\author{
Khaled Bensayah*, El-Ahcene Mahfoudi
}

CORIA UMR 6614 CNRS, Rouen, 76000, France

Corresponding Author Email: bensayahkha@yahoo.fr

https://doi.org/10.18280/ijht.360148

Received: 11 July 2017

Accepted: 2 January 2018

\section{Keywords:}

compressible flow, detached eddy simulation, SST model, nozzle, over-expanded, Shock

\begin{abstract}
Due to the large number of correlations and relationships between variables and the physical phenomena involved, compressible flow simulations become very difficult or impossible if all the necessary scales and mechanisms are included and solved. Several research efforts have been made toward a more accurate flow field predictions and the current study aims to add to that knowledge base by exploring the capability of Delayed Detached Eddy Simulation employing the SST turbulence model to simulate the transonic region of over-expanded nozzle with small radius of curvature. An analysis was made of the transonic flow in axisymmetric nozzle, the paper shows the potential for using DES turbulence model to identify important internal radial flow downstream the throat region, where most RANS models fail to predict with high accuracy and in detail the structure of the flow. With small radius of curvature, the sonic line begins upstream of the throat and ends downstream due to turning flow near the wall transonic region. Comparison of the computational results with experimental data and some developed prediction methods are presented and good agreements are obtained.
\end{abstract}

\section{INTRODUCTION}

The compressible fluid flow through a convergent-divergent nozzle undergoes a transition from subsonic to supersonic flow. The transition occurs in the throat region and separates the subsonic and the supersonic regimes. Even though there is a smooth physical transition from one regime to the other, the characteristics of the flow in these two regimes are quite different. In the subsonic region, the general equation that describes the flow is of the elliptical type, whereas in the supersonic region it is of the hyperbolic type. Methods of analyzing the flow in each of these two regimes have been well advanced and are generally available. Further difficulties arise, however, in treating the transonic region where both subsonic and supersonic flow exist and the method of analysis employed must be applicable to both types of flow [1].

Since the analysis of the supersonic flow region can, in general, be carried out independently of that of the subsonic region, calculations for the flow in the divergent portion of the nozzle are generally started from a known or calculated flow field in the throat. The problem of determining the transonic flow in a nozzle is thus of practical significance in the design of rocket nozzles, and methods have been devised to approximate the throat flow or to circumvent the problem completely. However, previous solutions to the transonic flow problem are most applicable to nozzle geometries with curvatures in the throat region such as are used in wind tunnels where nozzle length is not a prime consideration.

Rocket nozzles generally use a throat radius of curvature which is one-half to two times the throat radius. Under these conditions, the upstream boundary, particularly the entrance angle, may affect the flow pattern in the throat, and a realistic solution cannot be based solely on the wall curvature at the throat.

The throat flow region in nozzles has been widely studied [1-5], the ratio of throat radius of curvature to the throat radius

$\left(\mathrm{N}=\mathrm{r}_{\mathrm{c}} / \mathrm{r}_{\mathrm{t}}\right)$ is the important parameter that governs the isentropic flow in the transonic region of a supersonic nozzle. If this parameter is in order of one and greater the flow becomes one-dimensional and we obtain a gradual throat contour. The situation becomes very complicated if the radius of curvature decreases and two-dimensional effects become important in this case.

Existing two-dimensional flow theories [6-8] predict adequately the transonic flow field, but for nozzles with tighter radius of curvature $(\mathrm{N}<1)$, such as those found in some rocket engines, these theories do not apply.

To precisely predict turbulent flows at high speed would have a variety of important technological applications in the design of advanced hypersonic and supersonic space vehicles. Because of the wide range of scales displayed in these flows, all solved scales in the direct numerical simulations are impossible due the expensive cost of time and informatics tools. In addition, in complex confined geometries with large-eddy simulations applied to this kind of flows with the existing complex shock patterns are questionable [9]. The choice of modeling for the prediction of all the characteristics of compressible flows is therefore based on turbulence models known as the Wilcox k-w or the well-known SST model from Menter or the Reynolds stress modeling and this should remain true for the coming decades. It should be noted that many improvements in the modeling of Reynolds stresses in compressible turbulent flows that have been developed recently have not, for the most part, found their way into the prediction of aerodynamic flows of interest.

In this study, we try to treat a set of the most common tur- 
bulence model that the SST [12] and compare with a more sophisticated DES model which is the model which benefits from the advantage of the RANS [13-15] models near the walls and for do not consume more mesh and calculation time, and this is applied for quite complicated flows which present the internal flows in the radial direction near the wall, and for this comparison is harder and more persistent.

The turbulent model SST and DDES [16-18] based on SST model with compressibility correction are used to investigate the different flow characteristics. The detailed results of Mach number contour and many of results are presented to validate the numerical and turbulence modeling methods in the transonic region of supersonic flow, these results are also compared to some theories [6-8], unsteady method of characteristics has also been widely used to calculate the flow-field which is detailed in references [9-10].

\section{TURBULENCE MODELS AND NUMERICAL CODE}

The use of hybrid RANS-DES approaches with the combination of Reynolds' Navier-Stokes (RANS) and LES approaches has become increasingly important in recent years. Each of the two methods has its own advantages and disadvantages. The RANS approach has a tendency to predict accurately and with low or moderate computational cost near-wall flows that are attached. On the other hand, LES that solve very small scales has a very high computation cost compared to the RANS method, but this method can predict more and more precisely the detail of flow especially with the transient aspect. This hybrid method that has been recognized and has attracted attention is the detached eddy simulation (DES), which was proposed by Spalart et al. [19] this method, has yielded encouraging results for a wide range of flow types with massive separations. The motivation behind this approach was to combine the best features of the RANS approach near boundary layer with the best of LES for resolving the large eddies [20-23]. Therefore, DES turbulence processing aims to predict separated flows on unlimited Reynolds numbers and at reasonable cost. This hybrid RANS/LES method can provide and give good results for separated flows with complex shock pattern in which instability is highly autonomous.

\subsection{The SST turbulence model}

$$
\begin{aligned}
& \frac{\partial \rho k}{\partial t}+\frac{\partial}{\partial x_{j}}\left(\rho u_{j} k-\left(\mu+\sigma_{k} \mu_{t}\right) \frac{\partial k}{\partial x_{j}}\right)=\tau_{i j} S_{i j} \\
& +\left(1-F_{1}\right) \overline{p^{\prime \prime} d^{\prime \prime}}-\left(1+\alpha_{1} M_{t}^{2}\left(1-F_{1}\right)\right) \rho \beta^{*} k \omega
\end{aligned}
$$

$$
\begin{aligned}
& \frac{\partial \rho \omega}{\partial t}+\frac{\partial}{\partial x_{j}}\left(\rho u_{j} \omega-\left(\mu+\sigma_{\omega} \mu_{t}\right) \frac{\partial \omega}{\partial x_{j}}\right)=\gamma \frac{\omega}{k} \tau_{i j} \\
& -\rho \beta \omega^{2}-\left(1-F_{1}\right) \overline{p^{\prime \prime} d^{\prime \prime}} / \nu_{t}+\left(1-F_{1}\right) \alpha_{1} \rho M_{t}^{2} \beta^{*} \omega^{2} \\
& +2\left(1-F_{1}\right) \frac{\rho \sigma_{\omega_{2}}}{\omega} \frac{\partial k}{\partial x_{j}} \frac{\partial \omega}{\partial x_{j}}
\end{aligned}
$$

where $\tau_{i j}=2 \mu_{t}\left(S_{i j}-S_{k k} \delta_{i j} / 3\right)-2 \rho k \delta_{i j} / 3$ is the Reynolds stress tensor modeled by the Boussinesq eddy-viscosity hypothesis; $S_{i j}$ is the strain rate defined as $\left(\partial u_{i} / \partial x_{j}+\partial u_{j} / \partial x_{i}\right) / 2$; the con- $\operatorname{stant} \beta^{*}=0.09 ; M_{t}$ is the turbulent Mach number which is defined as $\sqrt{2 k} / a$ where $a$ is the speed of sound; the pressure dilatation term is $\overline{p^{\prime \prime} d^{\prime \prime}}=-\alpha_{2} \tau_{i j} \partial u_{i} / \partial x_{j} M_{t}^{2}+\alpha_{3} \rho \beta^{*} k \omega M_{t}^{2}$. The closure coefficients for the compressible corrections are: $\alpha_{1}=1.0, \alpha_{2}=0.4$, $\alpha_{3}=0.2$. The blending function $F_{1}$ is defined as:

$F_{1}=\tanh \left\{\left[\min \left(\max \left(\frac{\sqrt{k}}{0.09 \omega d} ; \frac{500 v}{d^{2} \omega}\right) ; \frac{4 \rho \sigma_{\omega 2} k}{C D_{k \omega} d^{2}}\right)\right]\right\}$

and the cross diffusion $C D_{k \omega}=\max \left(2 \rho \sigma_{\omega 2} / \omega \cdot \partial k / \partial x_{j} \partial \omega / \partial x_{j} ; 10^{-10}\right) ; \mathrm{d}$ is the distance from the nearest wall. Other constants are calculated from $\Phi=F_{1} \Phi_{1}+\left(1-F_{1}\right) \Phi_{2}$, where the $\Phi$ 's are the constants: $\sigma_{k 1}=0.85 \quad, \quad \sigma_{\omega 1}=0.5 \quad, \quad \beta_{1}=0.075 \quad, \quad \sigma_{k 2}=1.0$ $\gamma_{1}=\beta_{1} / \beta^{*}-\sigma_{\omega 1} k^{2} / \sqrt{\beta^{*}}=0.553, \sigma_{\omega 2}=0.856, \beta_{2}=0.0828$, $\gamma_{2}=\beta_{2} / \beta^{*}-\sigma_{\omega 2} k^{2} / \sqrt{\beta^{*}}=0.44$ and $\kappa=0.41$.

The eddy-viscosity of SST model is defined as:

$\mu_{t}=\min \left(\frac{\rho k}{\omega}, \frac{\rho a_{1} k}{\Omega F_{2}}\right)$

where $\Omega$ is the magnitude of the vorticity defined as $\Omega=\sqrt{2 w_{i j} w_{i j}}$ with $w_{i j}=\left(\partial u_{i} / \partial x_{j}-\partial u_{j} / \partial x_{i}\right) / 2$, denoting the rate of rotation tensor; $a_{1}=0.31$ and $F_{2}$ is included to prevent singular behavior in the free stream where goes to zero given by.

$$
F_{2}=\tanh \left\{\left(\max \left(2 \sqrt{k} / 0.09 \omega d, 500 v / d^{2} \omega\right)\right)^{2}\right\}
$$

\subsection{The hybrid rans/les turbulence model}

To construct a DES-type hybrid method based on two equations $k-\omega$ models, transformation is adopted for the destruction term in the turbulent kinetic energy transport equation. After introducing a length scale, this equation can be written as:

$$
\begin{aligned}
& \frac{\partial \rho k}{\partial t}+\frac{\partial}{\partial x_{j}}\left(\rho u_{j} k-\left(\mu+\sigma_{k} \mu_{t}\right) \frac{\partial k}{\partial x_{j}}\right)=\tau_{i j} S_{i j} \\
& +\left(1-F_{1}\right) \overline{p^{\prime \prime} d^{\prime \prime}}-\left(1+\alpha_{1} M_{t}^{2}\left(1-F_{1}\right)\right) \rho \beta^{*} k \omega \cdot F_{D E S}
\end{aligned}
$$

where $F_{D E S}$ is the hybrid function defined as

$$
F_{D E S}=\max \left[\frac{L_{t}}{C_{D E S} \Delta}\left(1-F_{S S T}\right), 1\right]
$$

With the length scale of turbulence $L_{t}$ is defined as $L_{t}=\sqrt{k} /\left(\beta^{*} \omega\right)$; the calibration constant is given by $C_{D E S}=C_{D E S}^{k-\omega} F_{1}+C_{D E S}^{k-\varepsilon}\left(1-F_{1}\right) ;$ Note that from the standpoint of DES only the $k-\varepsilon$ branch is important, since precisely this branch is active in the major part of the region where DES functions in LES mode here $C_{D E S}^{k-\varepsilon}=0.61$ and $C_{D E S}^{k-\omega}=0.78 . \Delta$ 
is the grid spacing defined as $\Delta=\max (\Delta x, \Delta y, \Delta z) ; F_{S S T}$ can be taken as $0, F_{1}$ or $F_{2}$. If $F_{S S T}=0$, the hybrid method reverts to a DES method of Strelets-type [20]. If $F_{S S T}=F_{1}$ or $F_{2}$, and this hybrid approach is called the Delayed Detached eddy simulation (DDES) [20-22]. Near wall, $\left(1-F_{S S T}\right)$ tends to zero due to the numerical properties of $F_{1}$ and $F_{2}$, and in this case the DDES will act in RANS mode. At the same time, $\left(1-F_{S S T}\right)$ takes a zero value outside the boundary layer and the DDES takes the original form of the Strelets model [20]. Therefore, DDES can therefore act in RANS mode near the wall without effect on the clustered grid scales. This means that DDES can delay switching from RANS mode to LES mode near the wall and this is due to the grid scales, particularly locally grids refined in the streamwise direction and spanwise direction for complex flow configurations. In this study the $F_{S S T}$ is taken equal to $F_{2}$.

\section{RESULTS AND DISCUSIONS}

The JPL (Jet Propulsion Laboratory) nozzle used had half-angles of convergence and divergence of $45^{\circ}$ and $15^{\circ}$, respectively. The ratio $\left(r_{c} / r_{t}\right)$ is equal to 0.625 [24-27].

In simulation, at $\mathrm{t}=0$ second the flow starts after the rupture of a diaphragm situated at the nozzle inlet that separates two regions of quiescent air. The stagnation pressure and temperature are at reservoir conditions, pressure $\mathrm{Pc}=4.82$ bar and temperature $\mathrm{Tc}=300 \mathrm{~K}$, whereas the low pressure is at atmospheric conditions, $\mathrm{Pa}=1$ bar and $\mathrm{Ta}=298 \mathrm{~K}$.

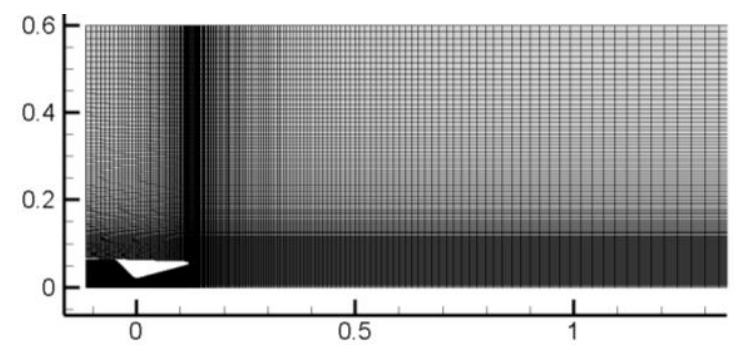

Figure 1. Mesh showing the external stretched far-field region

For calculations of expanded nozzle flows, the exit part domain of the exhaust jet of the nozzle must be included in the total calculation domain. The exhaust jet must be included as the flow separation within the nozzle strongly depends on the ambient pressure condition.

Numerical study has been performed by using home code, this code solves the three dimensional unsteady compressible Navier-Stokes equations on multiblock structured grid. The equations are discretized using a second order accurate upwind finite volume scheme and cell centered discretization. The viscous fluxes are discretized by Rho flux-difference splitting scheme. The numerical method was implemented in FORTRAN program in general curvilinear coordinates, and parallelization was introduced by partitioning the computational domain using the MPI libraries. The initial velocity field for the DES was obtained from a steady SST simulation. The code has the ability to impose specified velocity fluctuations on the initial velocity field to start the process. The SST steady solution provided an estimate of the initial turbulence velocity fluctuation. The initial time step used for the simulations was based on a time scale estimate for the integral length scales from the steady SST run and the condition that CFL $<1$ in the LES region. The near wall grid spacing was $1 \times 10^{-6} \mathrm{~m}$, the time step calculation was based on both velocity and speed of sound summation which can lead to a minimum time step, and it was taken equal to $5 \times 10-7 \mathrm{~s}$ based on courant number taken less than unity.

To ensure mesh-independent solution, the first grid point near the wall is located at $\mathrm{y}_{1}^{+}=\mathrm{u}_{\tau} \mathrm{d}_{\mathrm{w}} / v$ (where $\mathrm{u}_{\tau}$ is the friction velocity and $d_{w}$ the distance to the closet wall) to enable matching the fine grid clustering near the nozzle wall to the coarse grid in the regions far away from it. An extended grid refinement is obtained to ensure that the results are mesh-independent (Figure.2), and when the change of resolution between the subsequent mesh refinement steps has been considered negligible, the laminar viscous sublayer had to be resolved; this has been achieved by keeping the maximum nondimensional wall distance $\left(\mathrm{y}_{1}^{+}\right)$of the first point near the wall smaller than 1 (see Fig.2).

$5^{\circ}$ azimuthal slice along the nozzle modeled as multi-blocks, combining the nozzle, the free supersonic nozzle jet beginning from the outlet nozzle to the right exit domain, and the domain up the nozzle to the left exit domain. These three domains were discretized with nodes distributions in axial $\times$ radial $x$ circumferential directions as $220 \times 150 \times 4$, $180 \times 220 \times 4,50 \times 70 \times 4$ respectively for RANS model, and $290 \times 500 \times 4,240 \times 650 \times 4,100 \times 150 \times 4$ respectively for DES hybrid model.

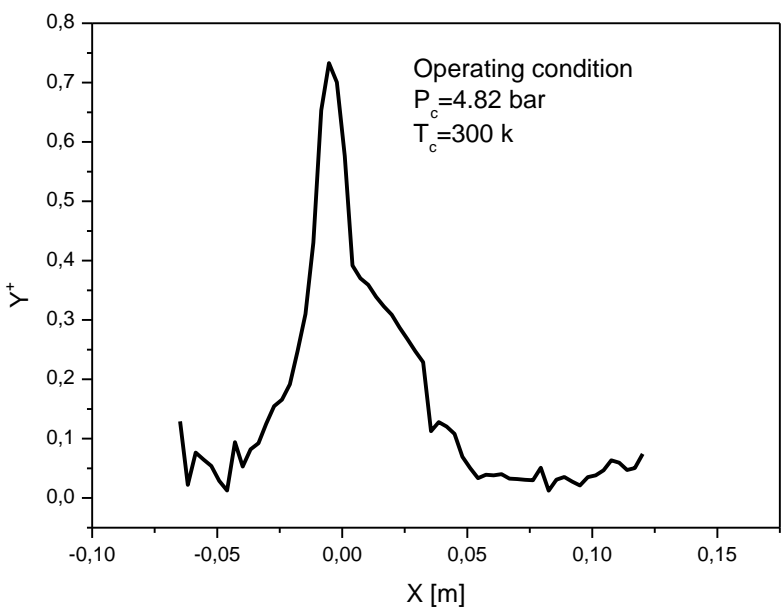

Figure 2. $\mathrm{y}_{1}^{+}$variation along wall nozzle

Figure 3 compares computational and experimental using the two turbulence models for the calculation of Mach number in the region which shows a more pronounced internal flow in the radial direction, the DES model calculates more precisely the Mach number, this is also shown in figure 4 with the shadowgraph, note that the author himself cited that the error of the measurements in this region can exceed $10 \%$ due to the difficulty of measuring the static pressure with great precision, in general the results are in good agreement with the measurements and show some detail of the attitude of the flow in the near wall region, we note here that the experimental Mach number is calculated for isentropic flow with $\mathrm{y}=1.4$ using the measured pressure according to the formula given by. We noted here that the wall Mach number is that of the first point near the wall and not exactly the wall position. 
$M=\sqrt{\frac{2}{\gamma-1}\left(\left(\frac{P_{C}}{P}\right)^{\frac{\gamma-1}{\gamma}}-1\right)}$

All next results will be considered done using the DES model.

To understand the fully behavior of the flow mechanism, a look into the contour plot of Mach number is essential.

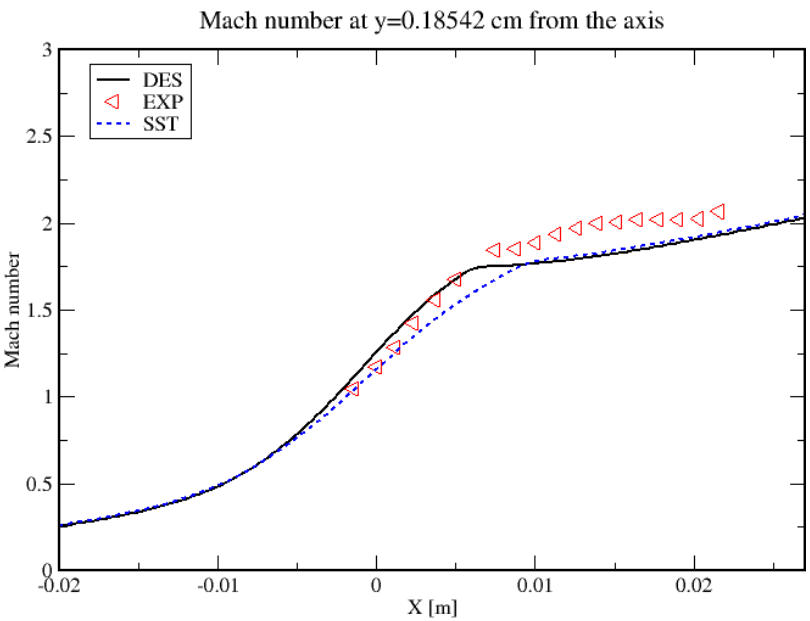

Figure 3. Comparison between SST and DES models

To ensure that numerical results would be meaningful, a close examination of Mach number field was done.

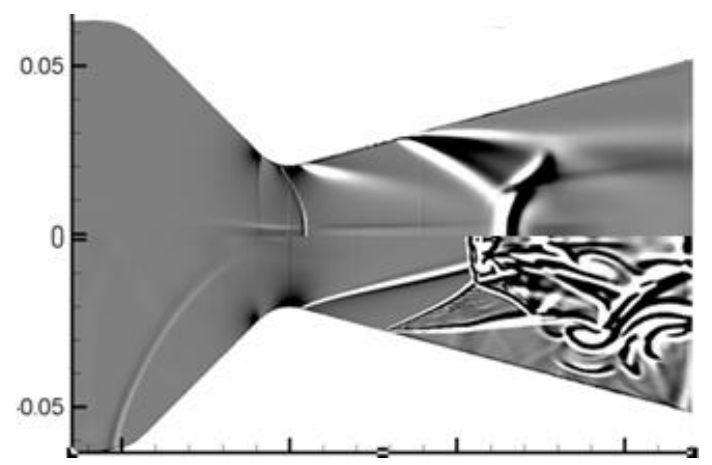

Figure 4. Shadowgraph (top, SST) and (Bottom, DES)

To validate the behavior of the flow numerically, a comparison was done with experimental data.

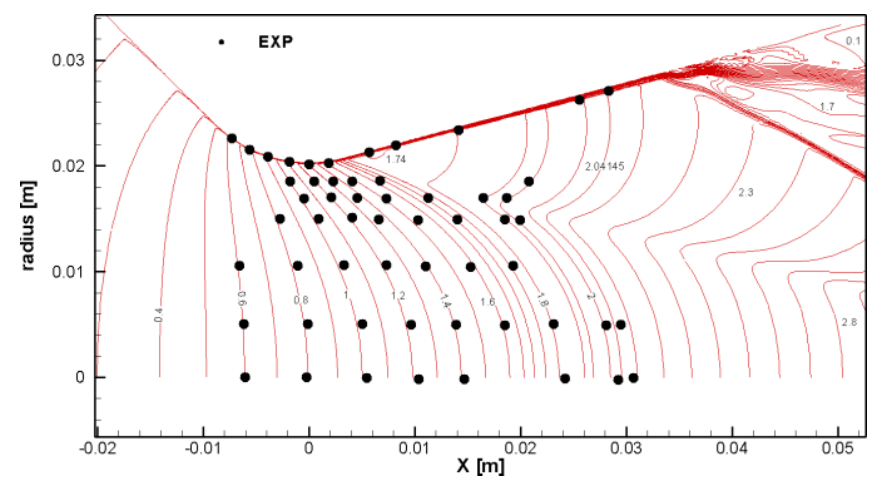

Figure 5. Mach number contour in the transonic region

Figure 5 shows the comparison between the Detached Eddy Simulation with the experiment of Cuffel et al [27], it can be seen that the calculations are in excellent agreement with the experiment, it can be seen clearly that the sonic line at the axis is located downstream the throat, but this sonic line is located just upstream of the throat location, this can be explained by the fact that the gas expands more rapidly along the wall nozzle than along the centerline, there are correspondingly large radial variations in the Mach number. This can also explained by the large pressure variations in the radial direction.

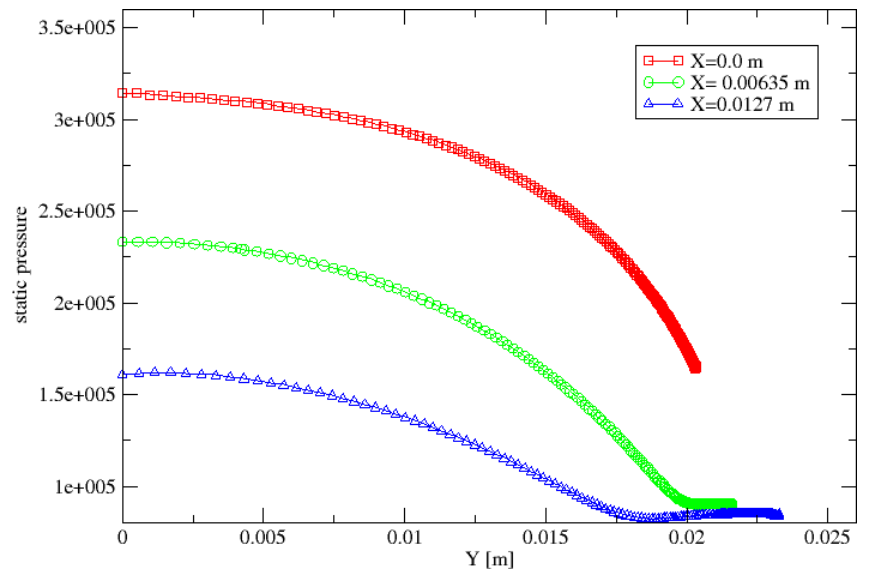

Figure 6. Calculated radial profiles of static pressure at the throat radius $(\mathrm{x}=0 \mathrm{~m})$ and at two locations downstream the throat.

Figure 6 shows the radial variation of the static pressure in the throat line at $(\mathrm{x}=0.0 \mathrm{~m})$ and two downstream locations when the internal flow shows some radials gradients in pressure and radial momentum.

The radial variation of pressure is important in transonic region; the values of pressure are two times greater those at the wall and the radial pressure gradients are about the same as the axial pressure gradients.

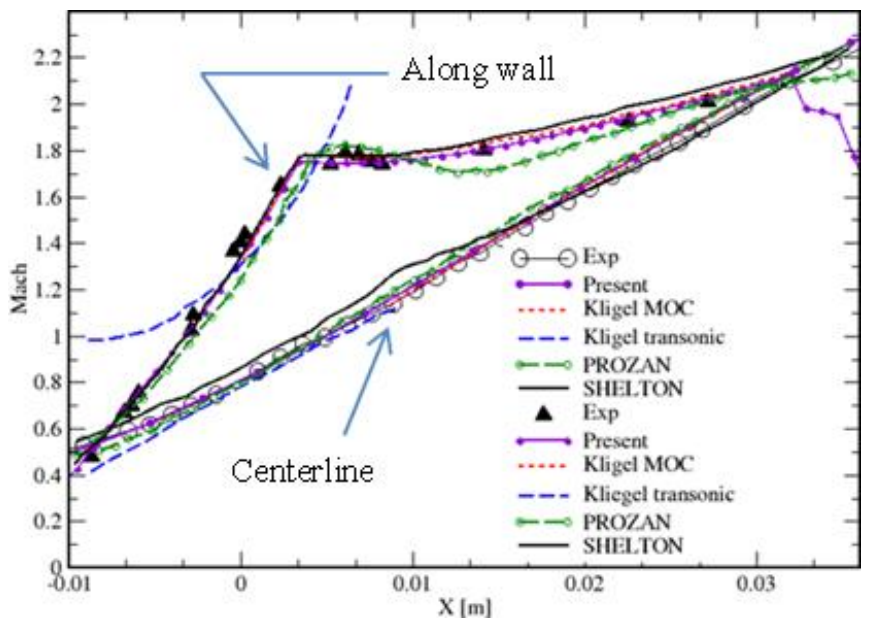

Figure 7. Comparison calculated of wall and centerline Mach number distributions, from transonic and method of characteristics (MOC) supersonic flow predictions, and the experimental results

The calculated Mach number is about 0.8 at the axis and 1.3 at the edge of the boundary layer. And this value rise to 1.76 just downstream of the throat (Figure 7).

The static pressure simulation data increases along the wall just downstream of the tangency between the circular arc throat and the divergent conical section shown in Figure. 1 is 
considered to be associated with a compressive turning of flow.

The obstinacy of the strong angular movement acquired by the flow in the region of the small radius of curvature throat can cause the flow to overturning so that the flow near the wall intensifies towards the downstream conical wall as indicated by the calculations.

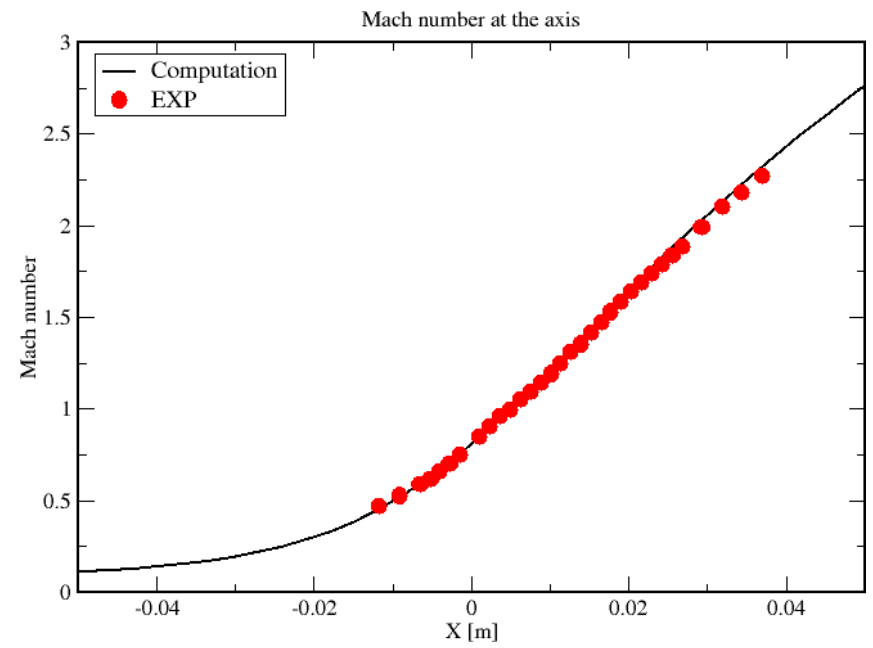

Figure 8. Calculated Mach number at the axis $\mathrm{y}=0 \mathrm{~m}$

The beginning region of convergence of the Mach lines, which is the beginning of the shock formation associated with the compression turning flow to become parallel to the wall, is evident in Figure 5 from the contour shape of the Mach number. The low oblique shock wave then forms and extends downstream, cutting the axis at $x=8 \mathrm{~cm}$. It is reasonable to assume that the flow is isentropic throughout the region.

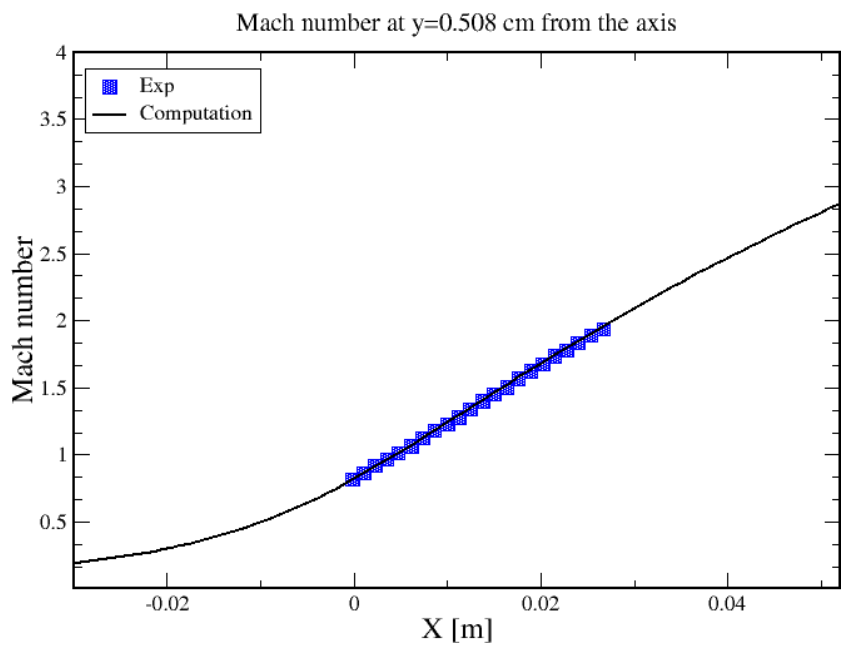

Figure 9. Calculated Mach number at $y=0.508 \mathrm{~cm}$ from the axis

Figures $8,9,10,11$ and 12 show the shape of the Mach number calculated in comparison with the experiment. The results are in very satisfactory agreement, very small differences are observed in the transonic region near the wall. At the first point near the wall nozzle the calculation is in excellent agreement, even the compression shock is well observed, and which results in a slight increase immediately followed by a decrease of the static pressure near the wall.
Various expansion techniques have been applied to describe the transonic flow field. All of these methods are essentially the same, being perturbations about the one-dimensional flow through the so-called normalized throat wall radius of curvature $\left(\mathrm{N}=\mathrm{r}_{\mathrm{c}} / \mathrm{r}_{\mathrm{t}}\right)$.

Hall [8] developed a transonic solution based on the small perturbation theory applying it to an irrotational, perfect gas.

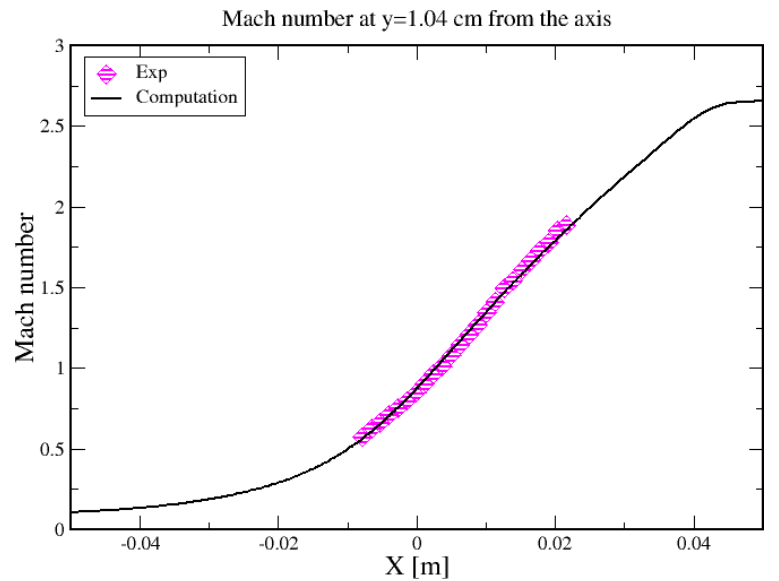

Figure 10. Calculated Mach number at $y=1.04 \mathrm{~cm}$ from the axis

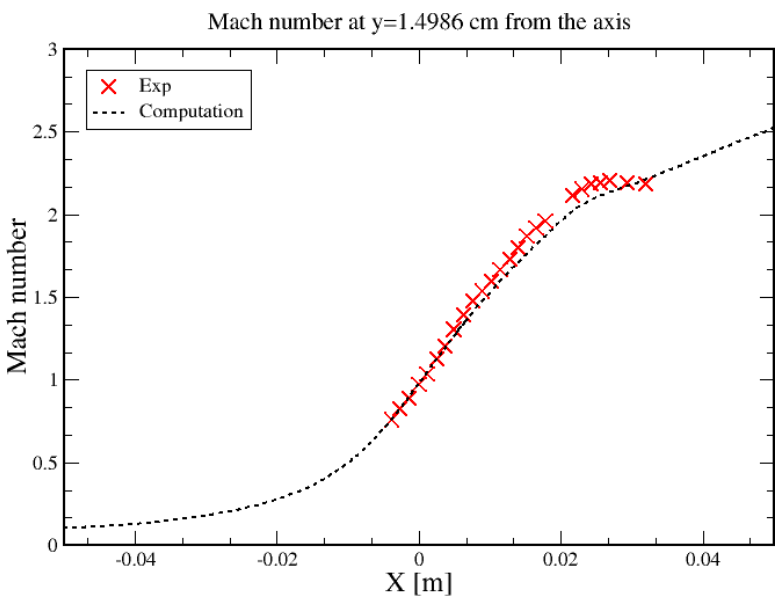

Figure 11. Calculated Mach number at $y=1.49 \mathrm{~cm}$ from the axis

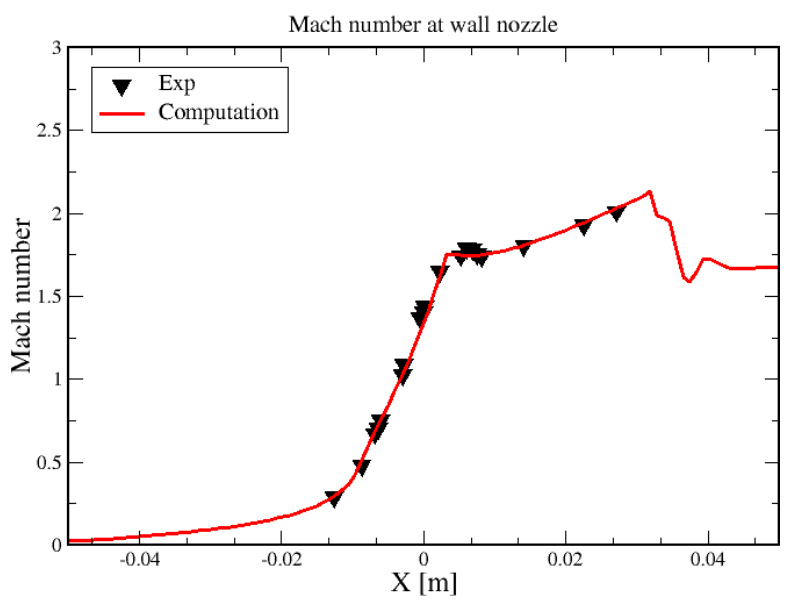

Figure 12. Calculated Mach number at first point near the wall nozzle 


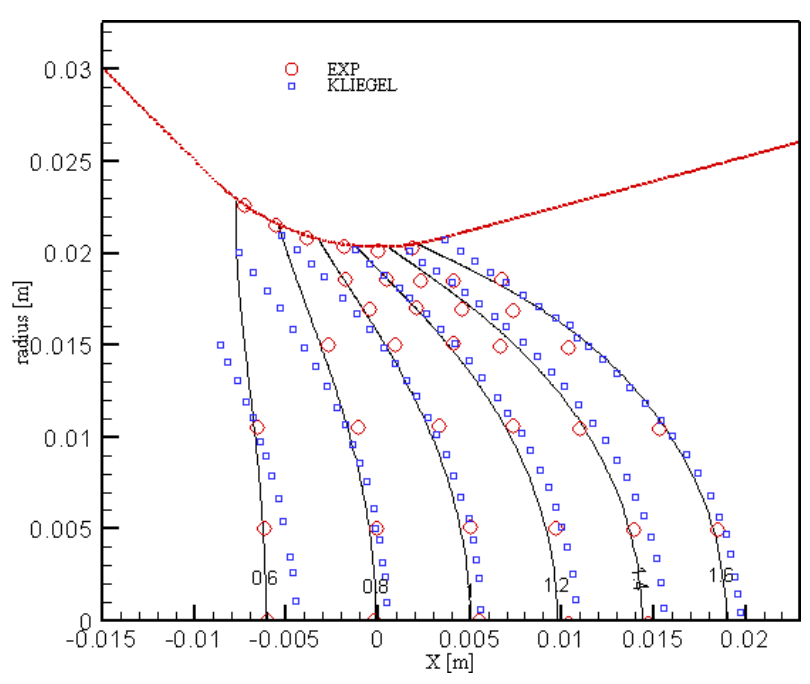

Figure 13. Transonic Mach number contour, comparison with theoretical predictions of Kliegel et al [28].

Using this toroïdal coordinate system and developing the solution in terms of $(1 / \mathrm{N}+1)$ instead of $(1 / \mathrm{N})$. In this case, the expansion parameter $(1 / \mathrm{N}+1)$ remains less than 1 for any value of the normalized wall radius of curvature $\mathrm{N}$.

One of the main advantages of the Kliegel and Levine method is its ability to produce good parabolic sound lines representing the two-dimensional aspect of the transonic solution independently of the value of $\mathrm{N}$. This is mainly due to the fact that the radius of Normalized Throat curvature does not have as much influence on the approach of Kliegel and Levine as it exists on the Hall methods.

As shown in figure 13, results of Kliegel et al are in good agreement in the throat plane, and reasonably good along the centerline, but deviates to a greater extent along the wall.

In the second method, the development of the transonic flow solution follows an inverse approach in that the boundary geometry is not specified but is obtained from the solution for a given velocity distribution along a suitable reference line. The usefulness of this approach depends on being able to specify, a priori, the velocity distribution that will yield the desired nozzle boundary. Hopkins and Hill [29], using Friedrichs'[30] formulation, applied this inverse method by empirically fitting the particular streamline to the nozzle wall contour, using a prescribed initial exponential velocity distribution along the centerline.

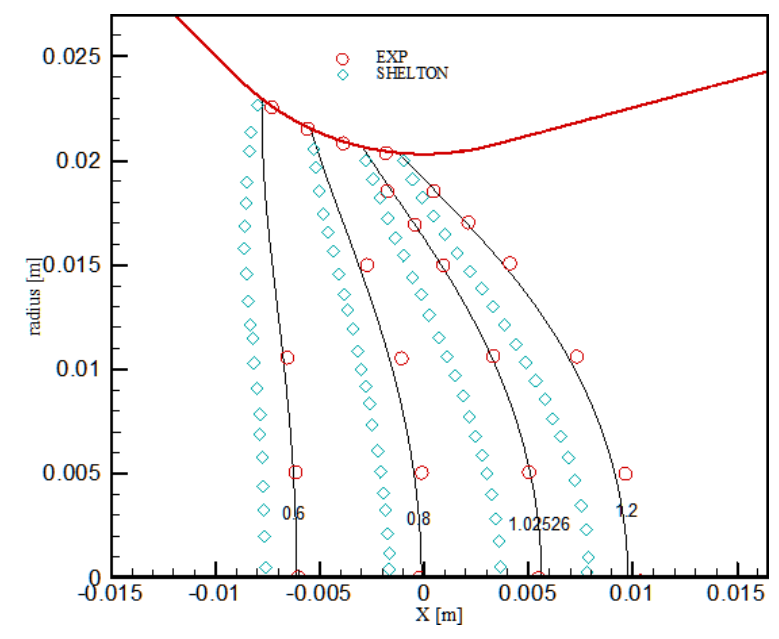

Figure 14. Transonic Mach number contour, comparison with theoretical predictions of Shelton et al [31]

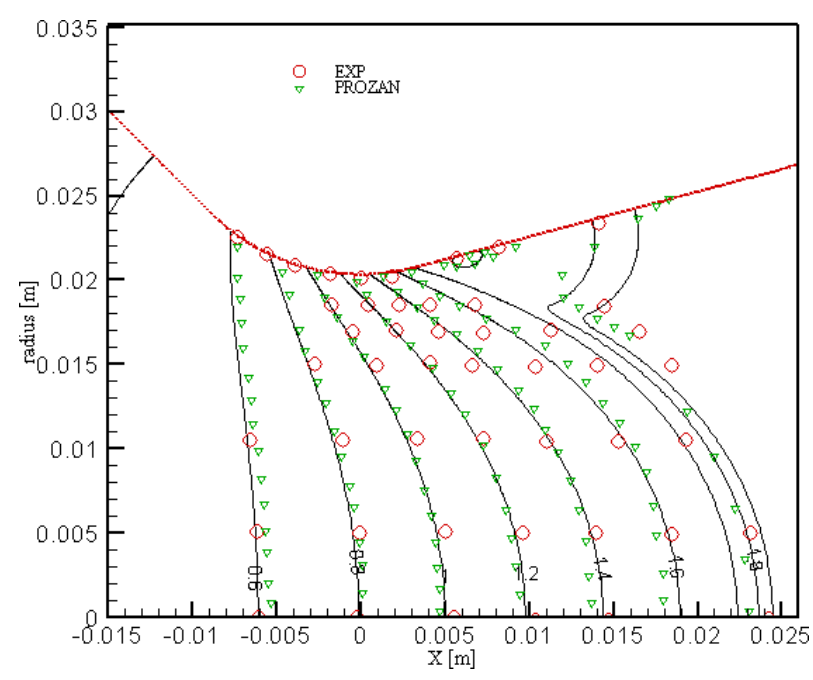

Figure 15. Transonic Mach number contour, comparison with theoretical predictions of Prozan et al [33]

A good streamline fit to the nozzle wall contour to 1.5 throat radii upstream of the throat was obtained by Shelton [31], also using Friedrichs [30] formulation, by choosing a third-order polynomial expression for the centerline velocity distribution and successively re-evaluating the polynomial coefficients. However, farther upstream the predicted contour deviates from the nozzle wall. This method agrees with the measurements along the wall in the transonic region (Figure. 14) but higher Mach numbers are predicted than are observed in the internal flow.

The method suggested by Crocco [32] was applied to the inviscid adiabatic nozzle flow, by numerically solving the time dependent motion equations (hyperbolic form) in subsonic, transonic and supersonic flow regions. The calculation of a summer carried out by Prozan [33] and the information on the technique are described by Saunders [34]. From an initially presc flow field considered as one-dimensional, the equilibrium solution is obtained as the limit of unsteady problem. The boundary conditions must be prescribed around the whole flow field, which necessitates an a priori specification of the mass flow rate. This prediction yields an inviscid flow coefficient of 0.990 , which is greater than the experimentally measured value of 0.985 . The distribution of the Mach number agrees with the experimental and simulation data results in the transonic region (Figure 15).

The predicted values along the wall are somewhat low just downstream of the sonic condition but agree with the data farther downstream, where the Mach number peaks and then decreases slightly. Prozan's prediction agrees well along the centerline (Figure 7).

Unlike the one-dimensional theory where they are simulated as straight vertical lines, they are found to be parabolic showing the two-dimensional aspect of the solution.

These theoretical methods are shown here for the simple reason of presenting the effort that has been deployed by researchers to find satisfactory results able to help the builders of the supersonic nozzles to take in hand a tool enabling them to carry out the design of the future supersonic engines, but in spite of all this, these methods have shown that it is incompatible to provide necessary information, such as the shock formation, and lateral charges causes by strong pressure gradients at the walls, such as the RSS (Restricted Shock Separation) who can damage seriously the nozzle and consequently the launching of spacecraft. 


\section{CONCLUSIONS}

The results shows that the use of the detached eddy simulation (DES) methodology presented here to simulate the flow behavior on a separated nozzle flow with small radius of curvature, focusing on the transonic flow field region is promising and needs further development.

The simulation data found on the axis and on a long range near the axis and on the wall are excellent, but a small area outside the boundary layer just downstream the throat of the nozzle, where the results are in good agreement with the experimental measurements, but which are not as excellent as the others and with what is expected with the use of a method LES or DES. This could be due to insufficient turbulence modeling in this region. That means that the simulation operates in LES mode without having the appropriate grid resolution. Because, that the optimal aims of researchers is to reduce the computational cost of the simulation with lower grid requirements and a subsequent lower restrictions on the maximum time step for explicit solvers, this could be remedied by refining the mesh, modifying the width of the filter, or adjusting the value of the DES turbulence model constants.

\section{ACKNOWLEDGMENTS}

MESRS (Ministry of Higher Education and Scientist Research of Algeria) is gratefully acknowledged for the grant $\left(\mathrm{N}^{\circ}\right.$ Y087134) for young scientist. The research reported herein was performed by the CRIHAN (Centre de Resource Informatique de Haute Normandie, Rouen, France) and JADE supercalculator CINES (Centre Informatique National de l'Enseignement Supérieur, Montpellier, France).

\section{REFERENCES}

[1] Yuan H, He Y. (2009). Transonic potential flows in a convergent divergent approximate nozzle. J. Math. Anal. Appl 353(2): 614-626. https://doi.org/10.1016/j.jmaa.2008.12.005

[2] Theodore von Karman. (1947). The similarity law of transonic flow. J. Math. And Phys 26: 182-190. https://doi.org/10.1002/sapm1947261182

[3] Kuz'min AG. (2002). Boundary Value Problems for Transonic Flow. John Wiley \& sons, West Sussex, 2002.

[4] Leith VSFO, Soviero PAO, Bastos-Netto D. (2005). Numerical evaluation of the flow inside the transonic nozzle of a direct-connect supersonic combustion research facility. 18th ICME, Nov. 6-11, Ouro Oreto, MG, Proceedings of COBEM. mtc-m16b.sid.inpe.br

[5] Wenxiang C. (2009). Applying numerical solution to analyze the performance of nozzles. ICEET'2009, 16-18 Oct. https://doi.org/10.1109/ICEET.2009.515

[6] Sauer R. (1947). General characteristics of the flow through nozzles at near critical speeds. NACA-TM-1147.

[7] Oswatitsch K, Rothstein W. (1949). Flow pattern in a converging-diverging nozzle. NACA-TM-1215.

[8] Hall IM. (1962). Transonic flow in two-dimensional and axially-symmetric nozzles. Quarterly Journal of Me- chanics and Applied Mathematics 15(4): 487-508. https://doi.org/10.1093/qjmam/15.4.487

[9] Thompson PA. (1972). Compressible Fluid Dynamics. McGraw-Hill Inc, US.

[10] Zucrow MJ, Hoffman JD. (1976). Gas Dynamics. Vol. I, John Wiley and Sons, New York.

[11] Lalmi D, Hadef R. (2017). Numerical study of the swirl direction effect at the turbulent diffusion flame characteristics, International Journal of Heat and Technology 35(1): 520-528. https://doi.org/10.18280/ijht.350308.

[12] Wilcox DC. (1988). Reassessment of the scale-determining equation for advanced turbulence models. AIAA Journal 26(11): 1299-1310. https://doi.org/10.2514/3.10041

[13] Cui WZ, Zhang XT, Li ZX, Li H, Liu Y. (2017). Three-dimensional numerical simulation of flow around combined pier based on detached eddy simulation at high Reynolds numbers, International Journal of Heat and Technology 35(1): 91-96. https://doi.org/10.18280/ijht.350112.

[14] Suzen YB, Hoffmann KA. (1998). Investigation of supersonic jet exhaust flow by one- and two-equation turbulence models. 36th AIAA Aerospace Sciences Meeting and Exhibit. https://doi.org/10.2514/6.1998-322

[15] Silton SI. (2005). Navier-Stokes computation for a spinning projectile from subsonic to supersonic. Journal of Spacecraft and Rockets, March 42(2): pp. 223-231. https://doi.org/10.2514/1.4175

[16] Menter FR, Kuntz M. (2003). A zonal SST-DES formulation. In: DES-Workshop. Technical University, St. Petersburg.

[17] Xiao ZX, Chen HX, Zhang YF, Huang JB, Fu S. (2006). Study of delayed-detached eddy simulation with weakly nonlinear turbulence model. Journal of Aircraft, September 43(5): pp. 1377-1385. https://doi.org/10.2514/1.20127.

[18] Fu S, Xiao ZX, Chen HX, Zhang YF, Huang JB. (2007). Simulation of wing-body junction flows with hybrid RANS/LES methods. Int. J. Heat Fluid Flow 28(6): 1379-1390. https://doi.org/10.1016/j.ijheatfluidflow.2007.05.007

[19] Spalart P, Jou WH, Strelets M, Allmaras SR. (1997). Comments on the feasibility of les for wings and on a hybrid RANS/LES approach. Proceedings of the 1st AFSOR International Conference on DNS/LES, edited by C. Liu and Z. Liu, Greyden, Columbus, OH, 1998: 137-147.

[20] Strelets M. (2001). Detached eddy simulation of massively separated flows. 39th Aerospace Sciences Meeting and Exhibit. https://doi.org/10.2514/6.2001-879

[21] Squires KD, Forsythe JR, Morton SA, Strang WZ, Wurtzler KE, Tomaro RF, Grismer MJ, Spalart PR. (2002). Progress on detached-eddy simulation of massively separated flows. 40th AIAA Aerospace Sciences Meeting \& Exhibit. https://doi.org/10.2514/6.2002-1021

[22] Forsythe JR, Squires KD, Wurtzler KE, Spalart PR. (2002). Detached-eddy simulation of fighter aircraft at high alpha. 40th AIAA Aerospace Sciences Meeting \& Exhibit. https://doi.org/10.2514/6.2002-591

[23] Deck S, Garnier E, Guillen P. (2002) Turbulence modelling applied to space launcher configurations. Journal of Turbulence 3(3): 1-21. https://doi.org/10.1088/1468-5248/3/1/057 
[24] Back LH, Massier PF, Gier HL. (1965). Comparison of measured and predicted flows through conical supersonic nozzles, with emphasis on the transonic region.

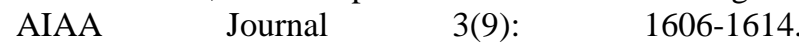
https://doi.org/10.2514/3.3216

[25] Back LH, Massier PF, Cuffel RF. (1967). Flow phenomena and convective heat transfer in a conical supersonic nozzle. Journal of Spacecraft and Rockets 4(8): 1040-1047. https://doi.org/10.2514/3.29015

[26] Back LH, Cuffel RF. (1966). Detection of oblique shocks in a conical nozzle with a circular-arc throat. AIAA Journal 4(12): 2219-2221. https://doi.org/10.2514/3.3881

[27] Cuffel RF, Back LH, Massier PF. (1968). Transonic flow field in a supersonic nozzle with small throat radius of curvature. AIAA paper 7(7):1364-1366. https://doi.org/10.2514/3.5349

[28] Kliegel JR, Levine JN. (1969). Transonic flow in small throat radius of curvature nozzles. AIAA Journal 7(7): 1375-1378. https://doi.org/10.2514/3.5355

[29] Hopkins DF, Hill DE. (1966). Effect of small radius of curvature on transonic flow in axisymmetric nozzles. AIAA Journal 4(8): 1337-1343. https://doi.org/10.2514/3.3674

[30] Friedrichs KO. (1944). Theoretical studies on the flow through nozzles and related problems. Rept. 82.1R, AMG-NYU 43, 1944, New York Univ.

[31] Shelton SV. (1967). Work done at the Jet Propulsion Laboratory, Pasadena, California, Unpublished report.

[32] Crocco L. (1965). A suggestion for the numerical solution of the steady Navier-Stokes equations. AIAA Journal 3(10): 1824-1832. https://doi.org/10.2514/3.3266

[33] Prozan RJ. (1968). Private communication, Lockheed Missiles and Space Co, Huntsville, Alabama.
[34] Saunders LM. (1966). Numerical solution of the flow field in the throat region of a nozzle. BSVD-P-66-TN-001 (NASA CR 82601), Brown Engineering Co, Huntsville, Alabama.

\section{NOMENCLATURE}

$k \quad$ turbulent kinetic energy, $\mathrm{m}^{2} / \mathrm{s}^{2}$

$\omega \quad$ turbulence eddy frequency, $1 / \mathrm{s}$

$\overline{p^{\prime \prime} d "} \quad$ pressure dilatation term,

$a_{1} \quad$ constant of the SST model

$S_{i j} \quad$ Reynolds strain, $1 / \mathrm{s}$

$\tau_{i j} \quad$ Reynolds stress tensor, $\mathrm{kg} / \mathrm{ms}^{2}$

$F_{1}, F_{2} \quad$ first and second blending function of the SST model

$C D_{k \omega} \quad$ cross diffusion coefficient

$r_{c} \quad$ radius of curvature, $\mathrm{m}$

$r \quad$ throat radius, $\mathrm{m}$

$x \quad$ axial distance, $\mathrm{m}$

$t \quad$ time, $\mathrm{s}$

$M \quad$ Mach number

$\Omega \quad$ vorticity, $1 / \mathrm{s}$

$\mu \quad$ dynamic viscosity of the fluid, $\mathrm{Pa}-\mathrm{s}$

$\mu_{t} \quad$ dynamic turbulence eddy viscosity, $\mathrm{Pa}-\mathrm{S}$

$C_{D E S} \quad$ constant of the DES-SST model

$F_{D E S} \quad$ switching function of the DES-SST model

$L_{t} \quad$ turbulence length scale, $\mathrm{m}$

$U_{j} \quad$ instantaneous velocity, $\mathrm{m} / \mathrm{s}$

$M_{t} \quad$ turbulent Mach number 